\title{
A HyperCard-based tool for studying cognitive processes in complex problem solving
}

\author{
STEVEN R. CARTER and DAVID A. WALSH \\ University of Southern California, Los Angeles, California
}

\begin{abstract}
A computer-based system for research in decision making, based on the concept of human problem solvers as builders and users of mental models, is described. This description is presented in three sections: (1) an overview of the behaviors that constitute the cornerstones of human problem solving in realistically complex domains; (2) a brief discussion of the substantive domain selected for studying these behaviors (financial problem solving), detailing the rationale behind its selection; and (3) a comprehensive description of the computerized decision-making system itself. The merits of using HyperCard in such research are discussed in terms of the efficacy and type of data collection engendered.
\end{abstract}

Research into judgment and decision making has been one of the fastest growing areas of psychology during the past two decades (Kahneman, 1991). However, the payoff in terms of our understanding of problem-solving processes in realistically complex situations has been distressingly slight. This relative lack of insight is due, in large part, to a lack of researchers involved in studying the role of knowledge in planning and guiding problem-solving behaviors.

Our understanding of problem solving has been based largely on normative theories, such as those associated with the von Neumann and Morgenstern expected utility theory and the Bayesian framework for reasoning under uncertainty. Unfortunately, these theories have limited applicability to a great many complex, real-world problems. Emphasis must be placed on a person's mental representation of a problem, and on the processing of information in which that person is engaged while generating a solution, if we are going to gain any understanding of problem-solving processes in realistically complex cases. In the words of one recent reviewer, "The heavy reliance on mathematical models that de-emphasize mental representation and the processing of information obscures some fundamental stages of the decision process in realistically complex cases" (Hastie, 1991).

Complex problem solving is guided by a person's understanding of the domain of information that the problem at hand represents. Such understanding, which can be conceptualized as a mental model or schema, organizes and directs a person's selection and use of information in generating a solution, and it may therefore account for the majority of variance in problem-solving performance and efficiency in most cases.

This research was made possible by a grant from the National Institute of Aging (1R01008106) to David A. Walsh. Correspondence should be sent to Steven R. Carter, Department of Psychology, University of Southern California, Los Angeles, CA 90089-1061.
We believe that one reason for the relatively small number of studies of the knowledge-driven processes that underlie problem solving has been the lack of practical and efficient research methodologies. Essentially, a method is required that will allow the researcher to ask a question, and then record everything that the subject does or thinks while generating a solution. A major obstacle to achieving this goal has been the overwhelming complexity of the behaviors involved in such a process. One of the goals of our research was to develop a set of realistically complex decision-making tasks, based on naturally occurring issues, set within an experimental environment that would allow for the empirical analysis of the cognitive processes underlying problem solving. Ideally, these tasks should allow for programs of research into both the applicability of normative theories of choice behavior and the descriptive investigation of the cognitive processes underlying human performance. Using the HyperCard medium with Apple Macintosh computers, we have developed an experimental environment that successfully addresses the complexity of knowledge-driven problem solving by creating a context in which a comprehensive record of a person's overt information-processing behaviors can be recorded.

First, we will present a brief description and theoretical discussion of the behaviors that we think constitute the cornerstones of human problem solving in realistically complex domains. Next, we will present a brief description of the substantive domain selected for use in our study of these behaviors (financial problem solving), and the rationale behind its selection. Finally, we will provide a comprehensive description of the computerized decisionmaking system that we have developed, and the dependent measures derived from it. This will be accompanied by an example of one of the research tasks, along with a problem-solving process "map" that shows a procedural script for gathering and manipulating data in a manner that leads to a correct answer. 


\section{Complex Problem Solving}

In his schema theory, Rumelhart $(1976,1980)$ presents the concept of a script as a representation of procedural knowledge. More recently, schemas have been defined as "unconscious mental structures that underlie the molar aspects of human knowledge and skill" (Brewer, 1987). This definition of schemas is similar to other "network" models of memory. In both cases, elements of knowledge are organized in relation to one another on the basis of specific topics or information categories.

Many current theoretical models of problem solving share the idea that knowledge is the key mediator of such behavior (Anzai, 1987; Hershey, Walsh, Read, \& Chulef, 1990). Hershey et al.'s (1990) script-based informationprocessing model posits that specialized procedural algorithms for considering information in complex problemsolving situations are developed through experience. These algorithms, or scripts, serve to maximize information search and selection efficiency during problem solving, while at the same time they minimize cognitive effort. The present research is based on this model. In short, we feel that complex problem solving is guided by a person's understanding of the domain of information that the problem at hand represents. This understanding can be broken down into two dimensions: knowledge of what pieces of information are relevant to a particular problem, and knowledge of how these pieces of information are interrelated.

Such understanding, which can be conceptualized as a mental model or schema, organizes and directs a person's selection and use of information in generating a solution, and it will thus be a major source of variance in problemsolving performance and efficiency. Therefore, the key behaviors of interest in studying problem-solving individuals revolve around those individuals' invocation of mental models as they consider data and generate responses. These behaviors include the determination of the data that need to be analyzed, the consideration of these data, the generation of higher order pieces of data that need to be considered but are not immediately available, and the generation of a solution to the problem at hand. Issues such as the effects of expertise, aging, strategy choice, and training on problem solving can all be addressed through the analysis of these behaviors and the mental models that they are proposed to represent.

\section{Task Selection}

Hershey et al. (1990) have argued that task selection has a profound effect on the information search and selection strategies of problem solvers. They note that factors such as task complexity, novelty, and structure have all been shown to affect the type and amount of information that subjects use. Cognitively complex tasks may elicit problem-solving strategies that minimize search in order to promote the efficient use of limited cognitive resources (Huber, 1980; Payne, 1976; Simon, 1955, 1989). Novel tasks, such as the Tower of Hanoi (Egan \& Greeno, 1974; Glass, Holyoak, \& Santa, 1979), Hobbits and Orcs
(Greeno, 1974), Missionaries and Cannibals (Rubinstein, 1975), and the Poisoned Food Task (Arenberg, 1968), may force a subject into "weak" problem-solving behavior, owing to the elimination of the role of task- or domain-specific knowledge in performance (Anderson, 1987; Charness, 1982). Such weak behavior includes strategies such as means-ends analysis, working backward, and analogical reasoning. Finally, well-structured tasks may leave little or no room for individual differences in problem-solving strategies (Simon, 1973; Voss, Tyler, \& Yengo, 1983).

Since the goal of the present methodology was to elicit behaviors that would invoke a range of mental models for problem solving, we sought a task sufficiently complex to encourage search efficiency. However, we also wanted to draw from a substantive domain that was sufficiently ill defined to allow the application of unique mental models, yet sufficiently familiar that subjects would have some level of prior knowledge and experience. Finally, and perhaps most importantly, we wanted to draw from a domain in which problem-relevant information had to be secured from the environment, so that we could record the acquisition of information.

Financial planning tasks were selected as meeting these criteria. Such tasks are informationally complex; knowledge of the domain specifies what variables are relevant and how they must be considered and/or manipulated for one to arrive at a correct solution. Financial planning tasks are also ill defined; situation-specific characteristics of a particular problem may play an important role in determining which variables should be considered and how they can be combined for one to arrive at a solution. Financial planning tasks are content heavy; although classes of variables may be well known, parameter values must almost always be secured from the problem-solving environment. And of course, such tasks can be as complex as one wants to make them. A financial planning task can easily require an individual to integrate information from dozens, or even hundreds, of variables in order to generate a solution. In addition, financial planning tasks can be analyzed to derive a prescriptive sequence of "correct" steps by conducting a task analysis to identify the conceptual elements one needs to consider, as well as the computational procedures one must follow in order to reach a correct solution. These attributes combine to yield an easily reduced trove of dependent measures, which can be efficiently recorded in the HyperCard programming environment.

\section{THE HYPERCARD COMPLEX PROBLEM-SOLVING ENVIRONMENT}

\section{Administrative Components}

The research tool that we have developed with HyperCard is an interactive medium that presents a problem to the subject, provides whatever information and/or computational aids the subject requires, and measures all overt information-processing behaviors engaged in by the sub- 
ject as the subject works toward a solution. This is done via the two-phase method developed by Hershey et al. (1990). In Phase 1, the subject reads a problem scenario and specifies the information required to solve it. Problem scenarios are generally a description of a hypothetical individual who is facing a financial decision. These decisions always involve a choice of action. For example, should a particular investment be made? Should a person be saving more for retirement? Should housing be rented or purchased? In Phase 2, the subject is provided with the information that he or she has requested, and is directed to generate a solution.

More specifically, the computerized complex problemsolving environment that we have developed with HyperCard consists of several conceptual, procedural, and administrative components. At the administrative level, the system consists of three parts: (1) an introductory component designed to familiarize subjects with the Macintosh computer and HyperCard environments; (2) a training component that instructs subjects on the use of the functions and tools specific to the problem-solving tasks; and (3) the complex problem-solving tasks themselves.

The introductory component, referred to as the Environmental Demonstration, implements our desire to study subjects regardless of their level of computer literacy. As such, the Environmental Demonstration addresses issues such as identifying and using the mouse and keyboard components of the computer, various components of the generic HyperCard interface, and certain objects that are unique to the problem-solving set.

The training component, referred to as the Functions Training, implements our desire to familiarize the subjects with the tools being provided for their use during the problem-solving task. Each subject is presented with a problem scenario and taken through a step-by-step procedure that generates the "correct" solution. The Problem Demonstration procedure is interactive, requiring the subject to perform tasks in a directed fashion. These tasks include the accessing of root level information, the calculation of simple sums, and the generation of time- and return-based projections of future value. The problem scenario used in the Functions Training component is derived from the same substantive domain as that used in the final experimental task, thus requiring similar knowledge and inference structures. However, it also requires the use and generation of largely different sets of root and higher order pieces of information. For example, if the final complex task involved retirement planning, the training scenario might involve a housing decision.

Finally, the complex problem-solving task component implements our desire to measure the scope and effectiveness of the subject's mental model of the problem domain. As such, the complex problem-solving task requires the subject to specify a data set for consideration that is based on the subject's interpretation of the parameters specified in the problem scenario, to synthesize intermediate level variables as is necessary, and to generate a solution.

\section{The Testing Component}

The complex problem-solving tasks consist of five conceptual components: (1) the problem description; (2) the subject-defined information array, (3) the problem-domain information array, (4) an interactive database, and (5) the computational tools.

The problem descriptions, referred to as scenarios, describe the problem to be solved; Figure $1 \mathrm{~A}$ gives an example. All scenarios consist of a hypothetical situation involving a complex, real-world financial issue. Little or none of the information essential to solving the task is included in the scenario. Thus, the scenario serves to prompt the subject to invoke some model of the problem being presented by requiring the subject to define the problem space (i.e., to specify what pieces of data are required to generate a solution).

Prior to testing, an exhaustive library of related data (both relevant and nonrelavent) is generated. This is accomplished through task analysis and a pilot survey with sample scenarios. A domain-specific HyperCard database containing a virtual card for each of these pieces of information is then created, along with an Executive Information Index that contains a descriptive label for each card. Figure 1B gives an example of an information card.

In the current version of the problem-solving environment, the subject-defined data array is created by the experimenter on the basis of the information requested by the subject after reading the scenario. This is done by selecting the appropriate card labels from the Executive Information Index. The resulting array, referred to as the Main Information Index, contains descriptive labels for the information cards that correspond to the pieces of data requested by the subject. Figure $1 \mathrm{C}$ gives an example of a subject-defined Main Information Index. The subject can access the information that he or she has requested, one card at a time (see Figure 1B), by selecting the appropriate descriptive label from the personalized Main Information Index.

The interactive database, referred to as the Scratch Pad, allows the subject to save and view processed data at any time during the task (i.e., from any "location" within the problem-solving environment). Figure 1D shows the Scratch Pad as it would appear when accessed from the Current Monthly Rent information card. Some sample information has been entered as well. The Scratch Pad is designed to act as the subject's workspace, allowing the subject to create an even more reduced or synthesized database as he/she works toward a problem solution. Additionally, the Scratch Pad was seen as a necessary analogue to real-world implements of memory support, such as notes, receipts, etc. Such items can be referred to and manipulated during complex problem solving, as well as viewed while one is engaged in calculations. To enable this, the Scratch Pad appears in two forms. First, the Scratch Pad, as pictured in Figure 1E, may be accessed by the subject in the foreground of any card image. The subject may thus consolidate information from several 
(A)

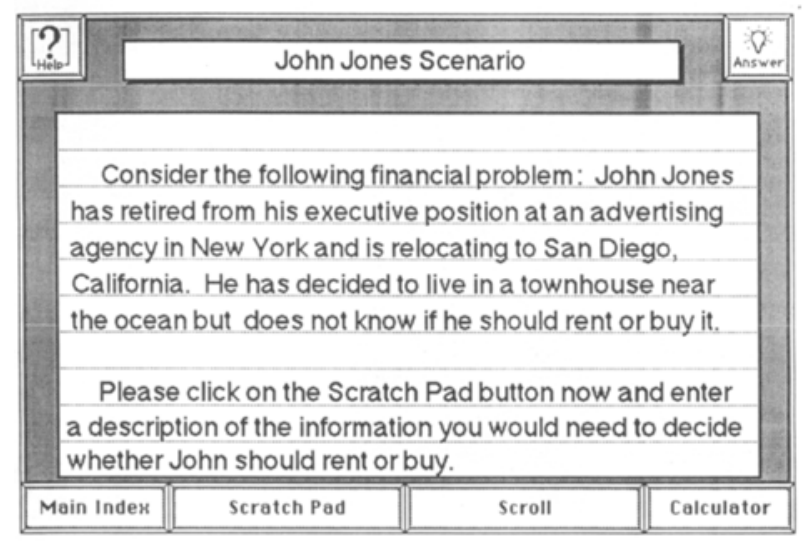

(C)

\begin{tabular}{||l|l||l||}
\hline [?] & \multicolumn{1}{|c|}{ Main Information Index } \\
\hline & $\begin{array}{l}\text { John Jones Scenario } \\
\text { Number of Years } \\
\text { Current Monthly Rent } \\
\text { Security Deposit } \\
\text { Rent Increases } \\
\text { Purchase Price } \\
\text { Down Payment } \\
\text { Mortgage Payments }\end{array}$ \\
\hline \hline Go Back & \multicolumn{1}{|c|}{ Scratch Pad } \\
\hline \hline
\end{tabular}

(E)

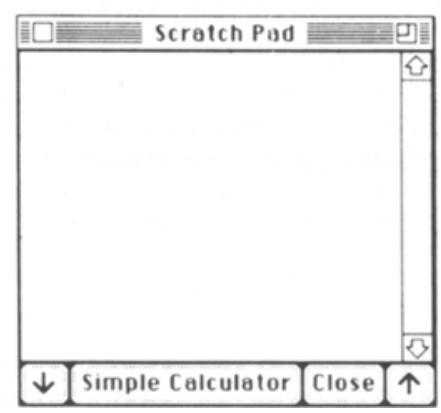

B)

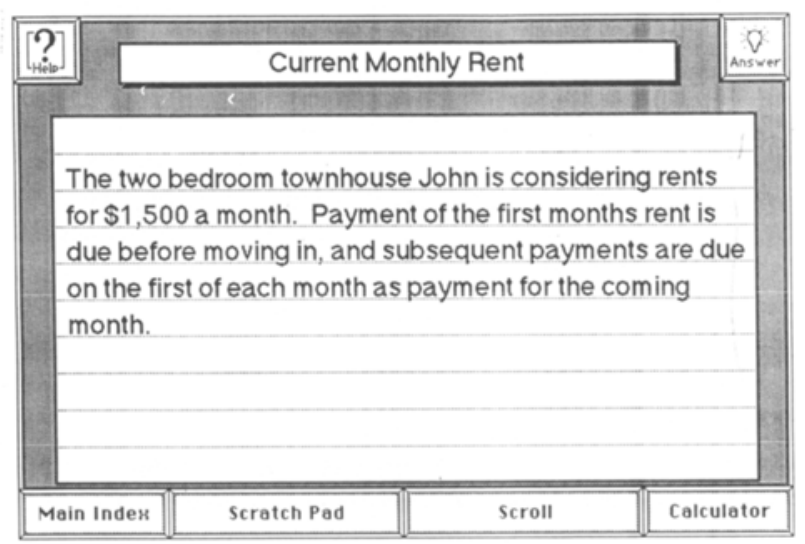

(D)

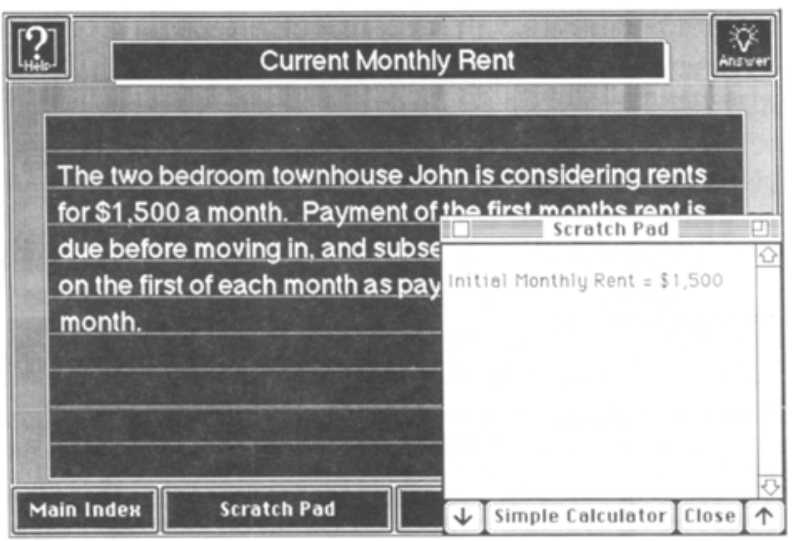

(†)

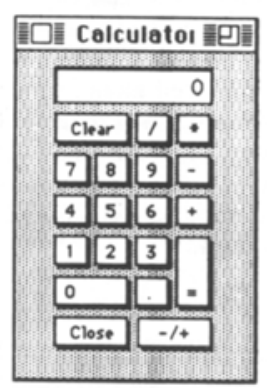


(G)

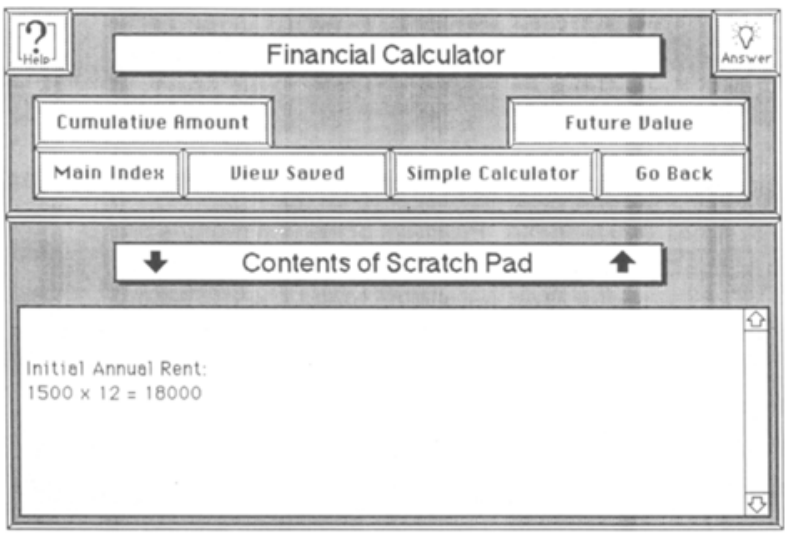

(H)

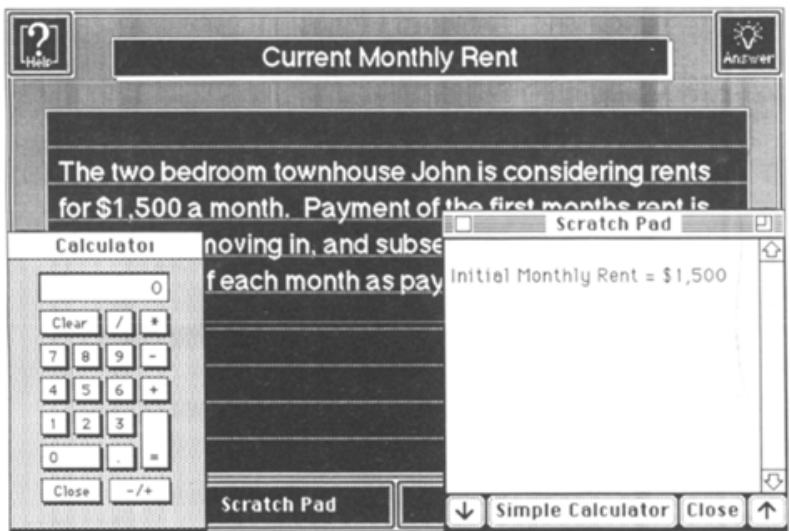

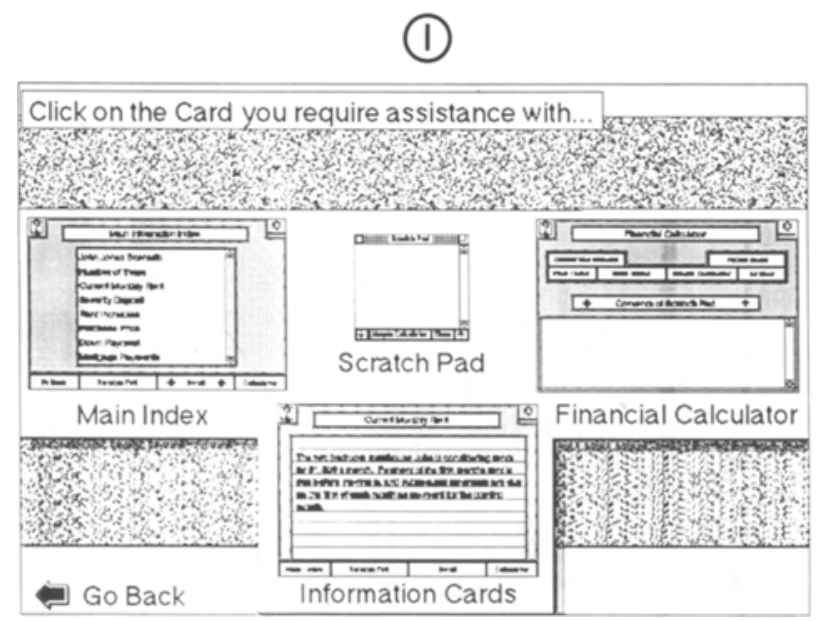

Figure 1. Card images from the HyperCard Complex Problem-Solving Behavior Measurement System. (A) Complex Problem Scenario card. This card describes the complex problem-solving task; it is the first card that the subject sees. (B) Data card. This is an example of the data cards available for the subject to use in generating a solution to the problem-solving task. (C) Main Information Index card. This card contains short labels describing the pieces of information requested by the subject. When clicked, each label in the center text field will take the subject to a data card containing the information described. (D) Scratch Pad and Data card. The Scratch Pad stack is accessed concurrenty with the Data stack. This allows information on cards in the "background" to be viewed while one uses the Scratch Pad. (E) Scratch Pad. The Scratch Pad, here pictured alone, consists primarily of an editable text field. (F) Simple Calculator. The Simple Calculator, here pictured alone, operates in the foreground of other card images. The Simple Calculator can be used with either the mouse or the keyboard. (G) Financial Calculator card. This is the primary computation tools component of the system. (H) Scratch Pad, Simple Calculator, and Data card. The Simple Calculator can be accessed from the Scratch Pad concurrently with the Data card stack. This allows information on cards in the background to be viewed while one uses the Simple Calculator. (I) General Help Index card. This is the first card of the General Help stack. Information pertaining to the function of various cards, fields, and buttons is provided within this stack.

sources into the Scratch Pad. Second, the contents of the Scratch Pad are transferred to a text box within the operational space of the main calculator component, shown in Figure 1G. The Scratch Pad contents box, located in the bottom half of the Financial Calculator card image, allows the subject to view stored pieces of information while using the various computational tools. This transfer of information is necessary, because in HyperCard the user can only access object functions specific to the foremost card image. Thus, to access the Scratch Pad proper would preclude the use of the computational tools contained within the Financial Calculator component.

Finally, the main computational tools component, already referred to as the Financial Calculator, allows the subject to perform a variety of simple and complex calculations. The Financial Calculator, shown in Figure 1G, provides three computational tools for the subject to choose from. The first is the simple Calculator, shown in Figure 1F. This tool is a direct analogue of a simple mathematical calculator, and is called into the foreground 
of the current card image in the same way as the Scratch $\mathrm{Pad}$ is accessed. The subject uses the simple Calculator either by clicking on the various screen buttons with the mouse, or by using their equivalents on the keyboard. The second tool is the Cumulative Amount function, which generates a projected cumulative value for some expense. When using this tool, the subject is prompted for three parameter values: (1) the time period for the projection in years, (2) the initial expense per annum, and (3) the rate at which the expense is expected to increase per annum. The third tool is the Future Value function, which generates a projected future value for some investment. When using this tool, the subject is prompted for seven parameter values: (1) the initial investment value, (2) the annual rate of return on this investment, (3) the initial annual amount of additional deposits made to this investment, (4) the annual rate of increase or decrease in said deposit, (5) the initial annual amount of withdrawals made from the investment, (6) the annual rate of increase or decrease in said withdrawals, and (7) the time period for the projection in years. Although the Cumulative Amount and Future Value functions can only be accessed from the Financial Calculator card, the simple Calculator can also be accessed from the Scratch Pad (see Figure 1H), making it readily available at any point during the task.

Subjects are provided with an on-line help component, for use in the event that any of the information provided in the training component of the system is forgotten. The General Help stack, accessible from any location within the system, provides an overview of the various card and button functions available to the subject. Figure 1I shows the first card of the General Help stack, from which the subject selects the image of the card about which he or she needs information. The General Help stack is thus a reminder of information contained within the Environment Demonstration and Functions Training components.

\section{Structure and Use of the \\ Problem-Solving Environment}

Now that we have described the conceptual components of the measurement system, we will describe more fully the functional components of this system, as well as the procedural aspects of using the system to generate a solution to a problem scenario. In general, it may be easiest to think of the current system as comprising several functional units, each of which is made up of a separate card or distinct HyperCard stack. Access to functional components within the environment is achieved either by opening a new card or stack that replaces the previous selection, or by opening a new stack that overlays the currently active stack.

The functional organization of the HyperCard stacks that constitute the problem-solving environment is depicted in Figure 2. Since the Environment Demonstration and Functions Training components are secondary to the experimental environment, we will describe only the five types of stacks that make up the Experimental Tasks and the Generic Functional Components.
A special Home stack contains most of the HyperTalk handlers and routines used by the system to collect data on a subject's information-processing activities. These measures will be discussed in the section on data collection. This stack also provides an executive "desk," providing the experimenter with an interface for setting up and running experimental sessions.

Figures 1A-1C show Problem Scenario, Data, and Main Information Index cards, respectively. It should be apparent that these cards are all based on the same background layout. A descriptive title appears at the top of each card, identifying the card content. Various action buttons are located on the card perimeter, and information relevant to the descriptive title appears in the card's center. These cards make up the functional component referred to as a Scenario stack. The two stack icons within the Experimental Tasks component of Figure 2 represent Scenario stacks. At the bottom right corner of the cards within these stacks, shown in Figures $1 \mathrm{~A}-1 \mathrm{C}$, the Calculator button offers access to the Financial Calculator. Clockwise from this button is the location for the Scroll button, which appears on cards that contain more text than can be displayed at once. Continuing clockwise, we find the Scratch Pad button, which offers access to the Scratch Pad stack; the Main Index button, which offers access to the Main Information Index card of the Scenario stack; the Help button, which offers access to the General Help stack; and finally, the Answer button, which will prompt the subject for a response and conclude the task.

The Main Information Index card (Figure 1C) offers two functional differences from the bulk of data cards: (1) Data cards are accessed by clicking on the labels contained within the central text field, and (2) the Main Index button at the bottom left becomes a "Go Back" button, allowing the subject to quickly return to a previous card if the Main Index button has been selected inadvertently.

The Financial Calculator has a slightly different layout from that of the Scenario stacks, as well as some additional action buttons. The Financial Calculator stack actually consists of two different types of cards: (1) the card containing the Financial Calculator itself, and (2) the Saved Calculation cards.

The Financial Calculator card, shown in Figure 1G, contains the calculator functions described in the discussion of the system's conceptual components. The buttons for activating the Cumulative Amount and Future Value functions are arrayed in the top half of the card. Beneath these are the Main Index, View Saved, Simple Calculator, and Go Back buttons. The Go Back button returns the user to the previous card if the Financial Calculator has been accessed inadvertently. The View Saved button allows the user to access previously conducted calculations. The Simple Calculator button allows the user to access the simple mathematical calculator function. And the Main Index button takes the user to the information index node. In the bottom half of the Financial Calculator card is a large text field. The contents of the Scratch Pad are entered into this field when the Financial Calculator card is opened. 


\section{Generic Functional Components}

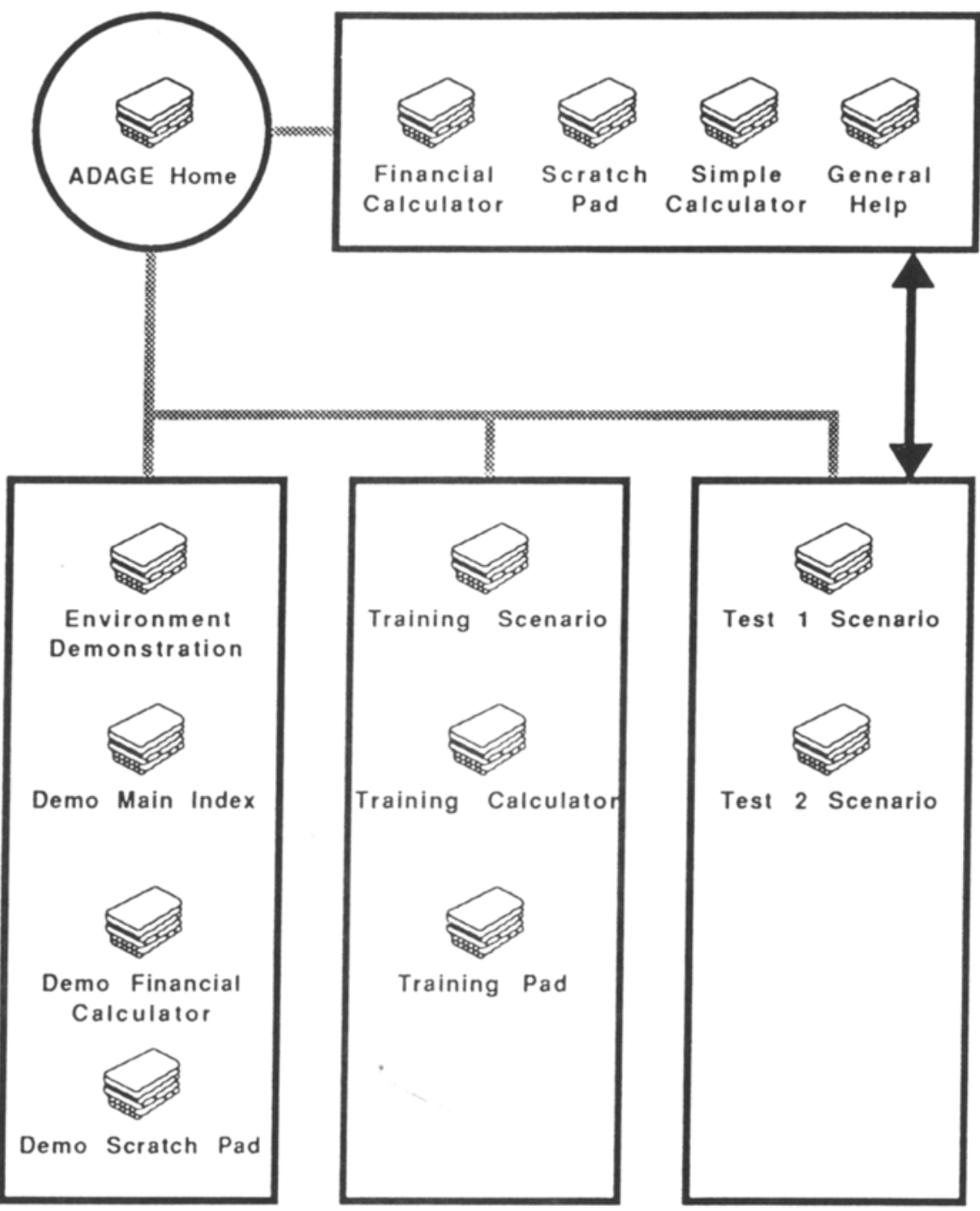

\section{Environment

\footnotetext{
Functional Dependence and/or

Experimenter Access$$
\begin{array}{r}
\text { Functional Dependence and/or } \\
\text { Experimenter Access }
\end{array}
$$

Functions

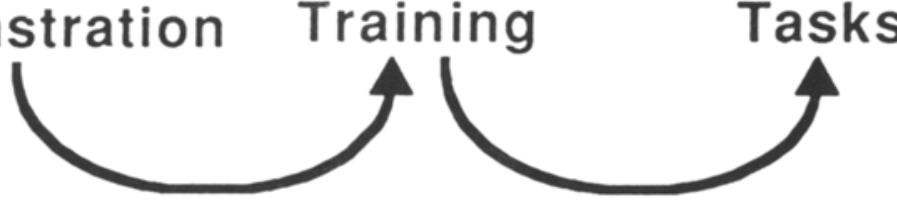

Experimental

\section{Tasks}

Subject Movement

Figure 2. Map showing the functional links between the various components of the HyperCard Complex Problem-Solving Behavior Measurement System. 
Above this field, in the title bar, are scroll buttons for controlling the area of text displayed.

The Contents of Scratch Pad field in the Financial Calculator card allows the user to activate the computational tools while simultaneously viewing the contents of the Scratch Pad. The actual Scratch Pad is a separate stack. A limitation in the current version of HyperCard is that one cannot access action buttons located on a stack appearing in the background (i.e., only one stack can be truly "active" when multiple stacks are in view). Thus, the contents of the Scratch Pad must be placed in the same stack as the calculator functions if the subject is to be allowed to both view and manipulate stored information while using the computational tools. The layout of the Financial Calculator card was shaped, in part, by this need.

When the user activates one of the complex calculation functions, the user is asked for a series of parameter values via dialog boxes (see Figure 3A). In the future, it would be desirable to redesign this card so that the Scratch Pad contents could occupy a more central location, allowing the action buttons to be placed along the bottom perimeter. This would enable a greater card layout consistency throughout the system.

In addition to requiring the user to supply various parameter values, both the complex and simple calculation functions require the user to provide a descriptive label for each of their calculations. After the test session, these descriptive labels are used by the experimenter to clarify the intent of ambiguous computations during data analysis. In the case of the Simple Calculator, these labels are entered into the Scratch Pad contents directly before the calculation information. In the case of the Cumulative Amount and Future Value functions, these labels are entered into the title bar of a new information card that is created to contain the description and results of the calculation conducted (see Figure 3B). Such cards are called Saved Calculation cards; the user may access them from the main Financial Calculator card by pressing on the View Previous button.

Saved Calculation cards are formatted much as the data cards accessed through the Main Information Index. The center of the card contains a text field with the information identified by the title bar. Along the bottom of the card, the primary action buttons Main Index, Scratch Pad, and Go Back are located consistently with respect to the rest of the system. Next to the Main Index button is the View Previous button. This button disappears when the last (earliest) saved calculation is reached. The Saved cards are accessed in a strictly linear fashion following a first in, last out architecture. Thus, previously conducted calculations can be thought of as a stack of information cards created by the subject, with the card that was created first located at the bottom of the stack. Above the Main Index button is the Delete button, which allows users to discard unwanted calculation cards from this stack.

The Scratch Pad is the final functional component used by a subject. This component is actually a stack with a single card that consists almost entirely of a text field that can be edited. Below this text field are four action but-

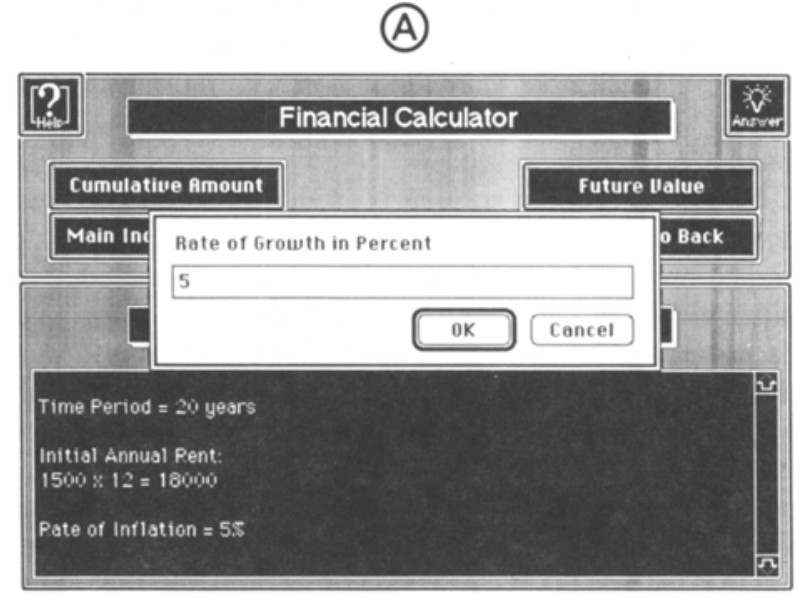

(B)

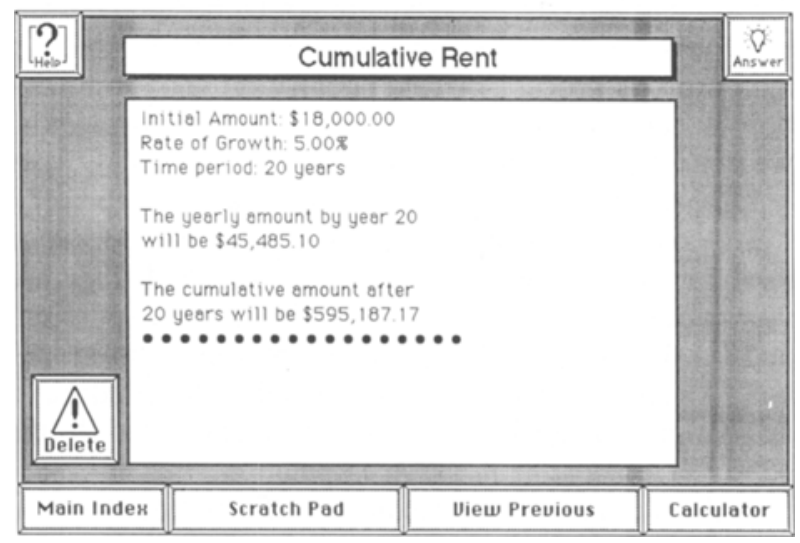

Figure 3. Additional Card images from the Computational Tools stack. (A) Financial Calculator. The complex computational tools provided within the financial calculator prompt the user for relevant information using dialog boxes as pictured here. (B) Saved Calculation card. This is an example of a data card created by the computational tools stack. It contains all of the information that the subject was prompted to provide, as well as the computational sums generated.

tons (see Figure 1E); the scroll buttons, which allow the user to control the area of text being displayed by the Scratch Pad; the Simple Calculator button, which accesses the Simple Calculator; and the Close button, which removes the Scratch Pad from use. As in the Financial Calculator, the result of any calculation conducted with the Simple Calculator is appended to its user-generated label and entered after the current Scratch Pad contents.

The most important difference between the Scratch Pad and the other functional components of the measurement system is the fact that the Scratch Pad is accessed concurrently with other elements. The primary role of the Scratch Pad is to provide the user with an immediately accessible medium for storing, retrieving, and viewing information. To facilitate storing information, the Scratch Pad was constructed to be small enough so that it could be operated while a data source was viewed in the background. Also, the Scratch Pad can be moved about the 
screen. This allows the user to achieve an unobstructed view of the information of interest on the background data source. The Simple Calculator is similarly movable.

\section{Data Collection}

Complex problem solving is guided by a person's understanding of the problem domain. This understanding can be broken down into two dimensions: knowledge of what pieces of information are relevant to a particular problem, and knowledge of how these pieces of information are interrelated. These two dimensions of understanding constitute a person's mental model of a problem. Thus, the key behaviors of interest in the study of problem solvers revolve about the invocation of mental models as they consider data and generate responses. These behaviors include (1) the selection of information that needs to be considered, (2) the analysis of this information, (3) the generation of higher order pieces of information that need to be considered but are not immediately available, and (4) the generation of a problem solution. To measure this process of data acquisition and manipulation by subjects as they work toward solving problems, we collect the following raw data:

1. A record of each piece of information requested.

2. A record of each piece of information viewed and/or used for computations: (a) the order in which they are viewed/used, (b) the length of time they are viewed/used, and (c) the number of times they are viewed/used.

3. A record of each information node calculated: (a) a subject-generated label for each computation, and (b) the substantive content of each computation.

4. A record of the aggregation of information; the contents of the Scratch Pad are recorded each time it is used, creating a record of entries and changes.

These data collectively provide a description of the subject's use and generation of information in solving the problem, referred to as a problem-solving process map (PSPM). Figure 4 shows an example of the PSPM for correctly deriving the cost of renting a house. At the bottom of this PSPM, we see the root level information that was considered. The root level consists of ecologically valid information that the subject has requested to solve the problem. Above these information nodes are the intermediary information nodes. These nodes are information that has been generated by the subject. As a labeling convention, the "order" of any piece of intermediary information is the highest level or order of information used to generate it, plus one. Thus, the information directly above the root-level information is called second-order information. With these problem-solving process maps, we can study a person's conceptual understanding of what information is relevant to solving the problem and how this information is interrelated. More specifically, the following dependent measures of subject's behavior are derived:

1. The root level information array considered relevant.

2. The number of higher order variables computed.

3. The number of information nodes viewed/generated.
4. The number of information nodes used in calculation or aggregation.

5. The subjective schema quality. Hershey et al. (1990) have noted that as expertise increases, the application of a problem schema becomes more efficient (i.e., there are fewer recursions through the selected data nodes). Extrapolating from this, we assume that the more confident one becomes with one's problem-solving procedure, the less rechecking of information one will engage in. Thus, we use the number of information nodes viewed or generated in comparison with the number of information nodes used in computations or decisions as an indicator of subjective schema quality.

6. Objective schema quality. Similarly to the preceding measure, the total number of data nodes considered and/or generated in comparison with the necessary number for the objectively correct solution is used as an indicator of objective schema quality.

These six indicators provide a description of a person's conceptual understanding of what information is relevant to the problem-solving task. An issue of much greater complexity is the subject's understanding of how these pieces of information are interrelated. This issue can also be addressed by analyzing a subject's PSPM. Each computation made is an indicator of the subject's understanding of how information at a lower level is combined/ interrelated to arrive at information on a higher level. Comparing a subject's PSPM with the "correct" PSPM (i.e., comparing the computations that the subject makes with the computations the subject should make) is one way of measuring the subject's understanding of how the considered information is interconnected. For example, the PSPM shown in Figure 4 consists of a hierarchical listing of all the "correct" computations necessary for determining the total cost of renting a house described by the parameter values given at the root level. We can evaluate the computations made by a subject in generating an estimate of the total cost of renting, given the same parameter values, on the basis of this "correct" PSPM.

In making these evaluations, the mistakes made by subjects in considering information become a complex and sensitive indicator of their understanding of how the information considered is interrelated. In general, there are two classes of mistakes that a subject might make. The first is errors of omission. This class of mistake comprises failures to request, generate, or consider relevant pieces of information. The second is errors of commission. This class of mistake comprises the request, generation, or consideration of incorrect or irrelevant pieces of information.

At higher levels of subject-generated information, errors of commission become an issue of some complexity. There are four ways in which a piece of information at or above the third order can be miscalculated and thus incorrect: (1) Incorrect information can be used; (2) correct but inadequate information can be used (i.e., two out of three relevant pieces of information are included); (3) "semantically" correct but "informationally" wrong information can be used (i.e., a correct second order vari- 


\section{Renting Cost PSPM}

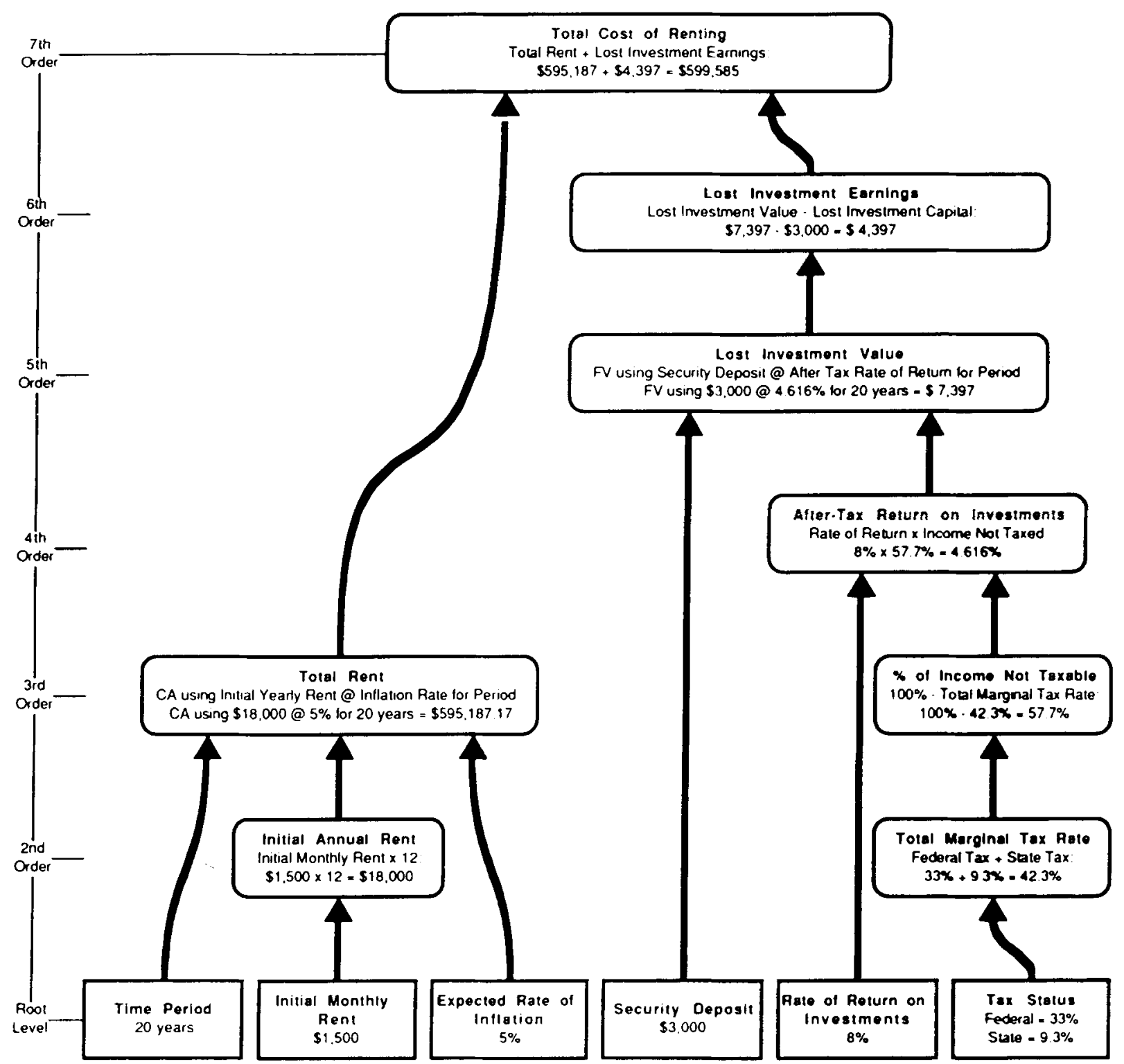

Figure 4. Problem-solving process map detailing the "correct" steps toward deriving the total cost of renting an apartment. Vertical axis to the left denotes the conceptual levels of information within the map. Arrows denote the flow of information "upward" from root to higher levels. Maps such as these are derived from the record of information-processing behaviors generated by the HyperCard Complex Problem-Solving Behavior Measurement System.

able could be used, but that piece of information could itself have been wrongly computed); and finally (4) adequate and correct information may be considered, but in a procedurally incorrect way (i.e., a relevant value might simply be added instead of subtracted).

For example, suppose that we wish to calculate the Investment Value that we would "lose" if we rented a house. Looking at the PSPM in Figure 4, we see that this is a fifth order piece of information, requiring a compu- tation based on a root level node (the security deposit required) and a fourth order node (the after-tax rate of return on investments). Computed correctly, as shown in Figure 4 , the lost investment value is $\$ 7,397$. Making the first type of error described above (using an incorrect piece of information), we might use the simple (root level) information regarding the rate of return on investments instead of its after-tax (third order) product. This would lead to a value of $\$ 13,983$ for the lost investment value. 
Making the second type of error described above (using insufficient information), we might not include the state taxes as part of the total taxes. This would lead to a value of $33 \%$ versus the correct value of $42.3 \%$ for total taxes. This error at the second order might then lead to the third type of error at the third order. Making the third type of error described above (using a semantically correct but informationally incorrect variable) at the third order, we might use our incorrect value of total taxes from the second order to compute the percentage of income not taxed. This would lead to a value of $67 \%$ versus the correct value of $57.7 \%$. Finally, making the fourth type of error (using correct input information but an incorrect procedure), we might multiply the initial annual rent by the rate of inflation, and then multiply the result by the number of years in the projection to arrive at an estimate of total rent. This computation would utilize all of the relevant information, but in a procedurally incorrect way.

To simplify scoring, we have further reduced the two classes of problem-solving process mistakes, referred to as data generation errors, into the two dimensions of data acquisition and procedural errors. Incorporating the issues described above, we arrive at the following additional subdivisions.

Data acquisition errors. (1) Omission: omit some relevant root level information; omit some relevant higher order piece of information. (2) Commission: consider an incorrect piece of information. (a) Conceptual: acquire and use a semantically wrong piece of information. (b) Content: acquire and use a semantically correct, but substantively wrong, piece of information. (3) Confabulation: make some piece of information up.

Procedural errors. (1) Omission: omit some necessary procedure in computing higher order information. (2) Commission: use wrong procedure in computing of information. (3) Confabulation: make some procedure up.

Data generation errors are indicators of both a subject's understanding of the interrelationships between pieces of information within the problem domain and the relevant data set. In other words, imperfections in a subject's knowledge of what data is relevant to a particular problem will be reflected in data acquisition errors, whereas imperfections in a subject's knowledge of how relevant data is interrelated within a specific problem domain will be reflected in procedural errors. Of course, these dimensions of understanding, and error, will be interrelated to various degrees as well.

\section{SUMMARY}

Knowledge is the key mediator of problem-solving behaviors in most complex situations encountered in the "real world." One's ability to deal with complex problems is based on one's knowledge of what information is relevant, and of how that information is interrelated. Such knowledge will shape and direct informationprocessing behavior that leads to the generation of a solution, and thus it will generally account for most of the variance in problem-solving efficiency and quality. With
Hypercard, we have developed a tool that allows for the study of problem solving as it is guided and directed by such knowledge in realistically complex situations.

HyperCard is ideal for this task for several reasons. First, HyperCard provides a powerful environment for presenting a variety of information to a subject and recording how the subject uses it. This, in concert with the use of problems drawn from domains of knowledge that require the subject to acquire immediately relevant information from the environment, makes HyperCard a powerful tool in itself for recording overt information-processing behaviors. Second, HyperCard provides a simple yet powerful scripting language (HyperTalk) that allows for the construction of different computational tools necessary for problem solving in a variety of substantive domains. Thus, HyperCard is flexible. Third, HyperCard is very user friendly both to the experimenter/developer and to the subject/user. As a self-compiling, objectoriented system, HyperCard and HyperTalk provide for the development of sophisticated tools with a minimum of computer knowledge. In addition, they provide for establishing a consistent and easy-to-understand point-andclick interface, within which subjects can quickly master movement from one component of the experimental environment to another.

In closing, we believe that three principles should be stressed with regard to the development of experimental tools for the study of complex problem solving. Most importantly, it is essential to incorporate into methodologies for the study of complex problem solving the element of requiring subjects to invoke their own mental models to define the problem space. Second, and to the above end, there is much to be gained by using problems drawn from domains that require the subject to acquire relevant information from the environment. Finally, emphasis must be placed on generating measures that allow the search for systematic differences in the range of behaviors that constitute the problem-solving process in complex cases.

In presenting our complex problem-solving environment, we hope that the principle on which it is based, as well as the specific components of its design, will be considered by others involved in the construction of similar tools. We hope further that this description will increase awareness of the benefits of using a computerized method in the study of complex cognitive processes, and that it will provide a resource for the future utilization of such tools in cognitive research.

\section{REFERENCES}

ANDERSON, J. R. (1987). Skill acquisition: Compilation of weak-method problem solutions. Psychological Review, 94, 192-210.

ARENBERG, D. (1968). Concept problem solving in young and old adults. Journal of Gerontology, 23, 279-282.

ANZAI, Y. (1987). Doing, understanding, and learning in problem solving. In D. Klahr, P. Langley, \& R. Neches (Eds.), Production system models of learning and development (pp. 55-97). Cambridge, MA: MIT Press.

BREWER, W. F. (1987). Schemas versus mental models in human mem- 
ory. In P. Morris (Ed.), Modelling cognition (pp. 187-198). Chichester, U.K.: Wiley.

Charness, N. (1982). Problem solving and aging: Evidence from semantically rich domains. Canadian Journal on Aging, 1, 21-28.

EGAN, D., Greeno, J. (1974). Theory of inductive learning: Knowledge acquired in concept identification, serial pattern learning, and problem solving. In L. Gregg (Ed.), Knowledge and cognition (pp. 4393). Hillsdale, NJ: Erlbaum.

Glass, A. L., Holyoak, K. J., Santa, J. L. (1979). Cognition. Reading, MA: Addison-Wesley.

Greeno, J. G. (1974). Hobbits and orcs: Acquisition of a sequential concept. Cognitive Psychology, 6, 270-292.

HASTIE, R. (1991). A review from a high place: The field of judgment and decision making as revealed in its current textbooks. Psychological Science, 2, 135-138.

Hershey, D. A., Walsh, D. A., Read, S. J., Chulef, A. S. (1990). The effects of expertise on financial problem solving: Evidence for goal-directed, problem-solving scripts. Organizational Behavior \& Human Decision Processes, 46, 77-101.

Hurer, O. (1980). The influence of some task variables on cognitive operations in an information-processing decision model. Acta Psychologica, 45, 187-196.

KAHNEmAN, D. (1991). Judgment and decision making: A personal view. Psychological Science, 2, 142-145.
PAyne, J. W. (1976). Task complexity and contingent processing in decision making: An information search and protocol analysis. Organizational Behavior \& Human Performance, 16, 366-387.

Rubinstein, M. F. (1975). Patterns of problem solving. Englewood Cliffs, NJ: Prentice-Hall.

RUMELHART, D. E. (1976). Understanding and summarizing brief stories. In D. LaBerge and S. J. Samuels (Eds.), Basic processes in reading: Perception and comprehension (pp. 265-333). Hillsdale, NJ: Erlbaum.

RUMElhart, D. E. (1980). Schemata: The building blocks of cognition. In R. J. Spiro, B. C. Bruce, \& W. F. Brewer (Eds.), Theoretical issues in reading comprehension (pp. 33-58). Hillsdale, NJ: Erlbaum.

Simon, H. A. (1955). A behavioral model of rational choice. Quarterly Joumal of Economics, 69, 99-118.

Simon, H. A. (1973). The structure of ill-structured problems. Artificial intelligence, 4, 181-201.

Simon, H. A. (1989). The scientist as problem solver. In D. Klahr \& K. Kotovsky (Eds.), Complex information processing: The impact of Herben A. Simon (pp. 375-398). Hillsdale, NJ: Erlbaum.

Voss, J. F., Tyler, S. W., Yengo, L. A. (1983). Individual differences in the solving of social science problems. In R. F. Dillon \& R. R. Schmeck (Eds.), Individual differences in cognition (pp. 205232). New York: Academic Press. 